Itinéraires Itinéraires

Littérature, textes, cultures

2017-2 | 2018

Féminismes quatrième génération

\title{
Anatomie de la salope. Des savoirs profanes autour du slutshaming, des slutwalks et de la culture du viol
}

Entretien avec Coline de Senarclens

Anatomy of the Slut. Secular Knowledge about Slutshaming, Slutwalks and the

Culture of Rape. Interview with Coline de Senarclens

Coline de Senarclens et Stéphanie Pahud

\section{(2) OpenEdition}

Journals

Édition électronique

URL : http://journals.openedition.org/itineraires/3848

DOI : $10.4000 /$ itineraires.3848

ISSN : 2427-920X

Éditeur

Pléiade

Référence électronique

Coline de Senarclens et Stéphanie Pahud, « Anatomie de la salope. Des savoirs profanes autour du slutshaming, des slutwalks et de la culture du viol », Itinéraires [En ligne], 2017-2 | 2018, mis en ligne le 10 mars 2018, consulté le 01 mai 2019. URL : http://journals.openedition.org/itineraires/3848; DOI : 10.4000/itineraires.3848

Ce document a été généré automatiquement le 1 mai 2019.

\section{cc) $(\mathrm{S} \odot$}

Itinéraires est mis à disposition selon les termes de la licence Creative Commons Attribution - Pas d'Utilisation Commerciale - Pas de Modification 4.0 International. 


\section{Anatomie de la salope. Des savoirs profanes autour du slutshaming, des slutwalks et de la culture du viol}

Entretien avec Coline de Senarclens

Anatomy of the Slut. Secular Knowledge about Slutshaming, Slutwalks and the Culture of Rape. Interview with Coline de Senarclens

Coline de Senarclens et Stéphanie Pahud

\section{NOTE DE L'ÉDITEUR}

Les nouveaux discours féministes sont généralement le fait de féministes jeunes, militantes et développant des sémiotiques particulières. Nous avons décidé de donner la parole à une militante suisse, Coline de Senarclens, cofondatrice de la Marche des Salopes Suisse - collectif né en 2012, devenu une association en $2014^{1}$-, et auteure de l'essai Salope ! paru en 2014 chez Hélice Hélas Éditeur, dans lequel elle décrit la stigmatisation sexuelle dont elle a été victime adolescente et la manière dont elle en a opéré la resignification en devenant militante. Coline de Senarclens est née en 1985, elle se définit elle-même comme « féministe, gauchiste, antiraciste ", elle est régulièrement invitée en tant que chroniqueuse dans une émission de la Radio Télévision suisse - Les Beaux parleurs - et s'investit dans la lutte pour l'autodétermination des femmes.

Coline de Senarclens, comment définissez-vous votre engagement?

Dans mon essai, je reviens sur ma propre expérience de stigmatisation : adolescente, j'étais la cible des attaques sexistes de mon école. J'ai d'abord été productrice d'un savoir profane, issu de vécus, de discussions, de ressentis, qui a peu à peu été reconnu comme un savoir expert par les institutions, les sphères académiques ou la presse. Je parle et j'écris en tant que témoin des violences qu'ont subi beaucoup de femmes autour de moi, en tant que survivante de stigmatisation et en tant que productrice d'un 
savoir à la croisée des sphères militantes et académiques. Une militante a écrit à propos de la Marche des Salopes : «It was a really empowering experience. » La militance offre un sentiment de légitimité et permet de se réapproprier l'espace public ainsi que la parole, grâce au témoignage. Il est difficile de dire si cette expérience marque durablement les comportements, mais il est incontestable, comme le théorise notamment Luca Greco (2014), qu'elle contribue à créer de nouvelles formes de savoir, développe la réflexivité et modifie nos rapports au genre et à la sexualité.

\section{Quel est le combat qui anime les slutwalks?}

Les discours médiatiques - et parfois les discours académiques - tendent à " déformer " l'esprit des slutwalks et à engendrer des confusions : les salopes ne descendent pas dans la rue pour "se réclamer d'une sexualité libérée", pour reprendre la formulation d'Élisabeth Mercier (2016), ou revendiquer la liberté de s'habiller comme elles le souhaitent; elles ne luttent pas pour leurs libertés individuelles, mais collectivement, contre les structures qui les oppressent, à savoir la culture du viol et le patriarcat. En réduisant les femmes à leur sexualité, et en distinguant les «bonnes» des "mauvaises", on cautionne une catégorie de femmes qui "prennent des risques » et qui «méritent » de se faire stigmatiser, harceler et violer. Derrière cette stigmatisation il y a des enjeux très clairs de rapport de pouvoir et de maintien de certains privilèges : qui peut se déplacer, où et quand; qui peut disposer de son corps; qui est en droit de s'exprimer et d'occuper les discours, notamment à propos de sa sexualité et de ses désirs; qui peut user de la violence, symbolique et physique, pour maintenir ces rapports de pouvoir ou punir ceux et celles qui dévient de leurs rôles assignés dans l'organigramme social (un organigramme hétérosexiste mais également raciste et classiste, nous ne le répéterons jamais assez).

Comment est née la Slutwalk Suisse?

Nous l'avons fondée notamment suite au témoignage de ma propre sœur à qui nous voulions collectivement offrir un lieu pour raconter son histoire dans une perspective militante, avec la conviction collective que son témoignage permettrait à d'autres de se libérer du sentiment de culpabilité de se réapproprier leur histoire. À l'époque, elle avait écrit ceci : "Le viol est une arme de domination masculine. Lorsqu'un homme viole une femme, il viole toutes les femmes, il assoit son pouvoir. Cela est dû au fait que la sexualité féminine ne s'appartient pas. Elle est garante de l'honneur, de la dignité de [...] son père, son frère, son mari, sa famille, son village, son pays, etc. Même la libération sexuelle des femmes n'a servi qu'à devenir un challenge: les femmes ne peuvent pas jouir, elles le doivent à leur partenaire, pour le rassurer... ${ }^{2} » C e$ témoignage a délié de nombreuses voix qui s'expriment dans le cadre de nos marches et de nos événements et font entendre des histoires proches, de violences, de honte, de culpabilité. Nous voulions un espace pour que nos amies, nos sœurs, nos collègues, nos (grand-)mères puissent témoigner de ces crimes ordinaires, et briser le silence.

En juin 2016 a eu lieu la cinquième Marche des Salopes à Genève. Comment l'événement se déroule-t-il, concrètement?

La Marche des Salopes est organisée par l'association Slutwalk Suisse; c'est une mobilisation contre les violences sexuelles, le slutshaming, la culpabilisation des victimes et la culture du viol. La dernière Marche a compté environ 500 militantes qui ont défilé entre la place de la Navigation dans le quartier des Pâquis et la place de Neuve devant le fameux parc des Bastions où règnent Calvin et les réformateurs. Elle $\mathrm{a}$ pris fin sur un moment de témoignage public durant lequel des femmes ont pu raconter 
les violences qu'elles avaient subies devant un parterre à l'écoute. L'association est désormais intégrée par des partenaires institutionnels tels que la ville et le canton de Genève, à des projets de lutte contre les violences faites aux femmes. Autrement dit, les Salopes ont gagné leur légitimité et ont fait bouger certaines normes.

Pourquoi vous concentrer sur les violences sexuelles?

Qu'il soit question de maternité, de sexualité, de mobilité géographique, de consommation d'alcool et de stupéfiants, ou de canons esthético-corporels, l'enjeu de la réappropriation du corps est central. Les débats actuels autour du harcèlement de rue qui poussent des hommes à déclarer : "J'ai le droit de dire à une femme qu'elle est belle ", montre parfaitement que le corps des femmes est encore considéré largement comme à disposition des hommes et que ces derniers passent pour être légitimes à les juger et à les commenter. Les comportements des femmes sont sujets à jugement, en particulier quand ils sortent des rôles traditionnels féminins. Le double standard dans tous les domaines permet de disqualifier les femmes qui tiennent des positions dirigeantes, font de la politique, ont de l'ambition... Mais il permet tout aussi bien à un mari de stigmatiser sa femme qui pourtant se soumet aux injonctions patriarcales parce qu'il souhaite marquer sa domination. Aucun comportement ne protège les femmes, ni contre les violences sexuelles, ni contre la stigmatisation. Ces violences agissent comme un outil coercitif d'assignation. En amont, il encourage les femmes à respecter les rôles traditionnels de genre. En aval, il excuse les auteurs de violences et disqualifie les victimes.

\section{D'où vient selon vous ce double standard que vous évoquez?}

Quand j'avais 8 ans environ, j'ai suivi un cours d'éducation sexuelle au cours duquel la formatrice a expliqué à l'ensemble de la classe que les garçons pensaient au sexe alors que les filles pensaient à l'amour. Cette déclaration n'a aucunement été déconstruite. Elle a été présentée comme une vérité générale, universelle. On m'a appris que j'étais faite pour aimer et non pour désirer, parce que j'étais une fille. De même que les enfants LGBT intègrent tôt que leur affectivité est "étrange », les filles intègrent que «leurs désirs font désordre " et surtout les dégradent; de même que les enfants LGBT constatent que les histoires qu'on leur raconte et les dessins animés qu'ils regardent ne présentent pas de personnages qui leur ressemblent, les filles évoluent de l'enfance à l'adolescence dans un système qui survisibilise et surlégitime la sexualité des hommes et qui invisibilise le désir féminin. Et alors que les garçons et les adolescents peuvent découvrir leur sexualité et leurs désirs légitimement, les filles sont menacées par le slutshaming. Par contre, le sentiment amoureux leur est imposé avec beaucoup d'insistance par une production culturelle également problématique, en ce qu'elle représente les fondements d'une dépendance affective. Il en résulte une confusion entre désir et affectivité, et surtout une difficulté à penser sa libido, et donc à savoir ce qu'on veut. Considérées très tôt comme objets et non comme sujets du désir, les femmes disposent de peu de ressources culturelles, de mots, d'espaces de réflexion pour penser leur sexualité et leur libido. Or, c'est en étant capable de savoir ce qu'on veut et ce qu'on aime qu'on est capable de savoir ce qu'on ne désire pas. C'est la construction identitaire même qui est touchée par ce double standard, assisté par une institution scolaire sexiste et présent dans de nombreux discours. La structure oppressive du double standard et des violences symboliques qui en découlent biaise la vision des sexualités. 


\section{Qu'est-ce que le slutshaming?}

Le slutshaming, de l'anglais slut, "salope » et du verbe to shame, "faire honte ", est une violence structurelle envers les femmes et un outil social de maintien des rôles de genre. Basé sur un double standard sexiste qui valorise la sexualité masculine et dégrade celle des femmes, il mobilise la sexualité prêtée ou avérée d'une femme, cis- ou transgenre (ou d'un homme dans le cadre de l'homo et de la transphobie) pour la stigmatiser, la disqualifier ou justifier des violences à son égard. Comme l'objectivation des femmes et de leur corps tend à les réduire systématiquement à leur sexualité, plus ou moins tous les aspects de la vie des femmes finissent par prêter le flanc au slutshaming. La consommation d'alcool, le port de maquillage, la possession d'une belle voiture ${ }^{3}$, une position hiérarchique élevée sur le marché du travail ou en politique... Aucune femme n'est à l'abri de se faire slutshamer, aucun comportement ne garantit son évitement. Les femmes sont stigmatisées parce qu'elles dévient des normes de genre qui leur sont imposées, que ce soit sur le plan sexuel, professionnel, au niveau de leur mobilité géographique, de leur consommation d'alcool ou de drogue, de leur refus de la parentalité ou parce qu'elles allaitent en public. De la même manière que l'hétéronormativité met les enfants LGBT en porte à faux vis-à-vis de leurs désirs et de leur affectivité, le slutshaming remet en question la légitimité des filles et des femmes à désirer et à explorer leur sexualité et leur libido. Mais n'importe quelle femme peut être la cible d'attaques stigmatisantes, notamment suite à un viol, ou parce qu'on cherche à la discréditer. Le slutshaming disqualifie les femmes, et en les privant de crédibilité, les empêche de créer du discours à propos d'elles-mêmes. Ce faisant, il les silencie. L'existence des femmes stigmatisées est salie, entachée, et la seule manière de la laver, c'est de cesser d'exister. Les (tentatives de) suicides et les phobies scolaires (disparition de l'espace d'enseignement) liées au harcèlement le montrent bien. La Marche des Salopes tente de réhabiliter l'idée qu'aucune sexualité n'est illégitime du moment qu'elle est mutuellement consentie, qu'aucune femme ne mérite d'être stigmatisée et que cette stigmatisation participe d'un système de domination menaçant toutes les femmes.

Slutshaming veut dire en français «stigmatisation des salopes ». Mais comment faut-il comprendre ce terme de salope?

Sur l'une des pancartes réalisées lors d'une soirée de préparation ouverte à toutes et tous, et lors de laquelle les militantees, ensemble, ont travaillé à faire corps pour lutter contre la culture du viol, on pouvait lire : «Et le patriarcat créa la Salope pour violer tranquille. » Le patriarcat a construit un concept, la salope, qui permet de stigmatiser et objectiver les femmes qui ne respectent pas ses règles. Comme je l'ai écrit dans un article consacré à l'association Aspasie (Senarclens 2012), de la même manière qu'il est très difficile de définir une prostituée et a fortiori une putain, rassembler un ensemble de facteurs qui font la salope est impossible, tant l'usage du mot varie et répond à des comportements ou des pratiques non homogènes. Le terme est parfois associé aux «femmes de mauvaise vie », dont la sexualité est jugée décadente, qui auraient «trop » de partenaires sexuelle's, apprécient trop le sexe, ou en font commerce. Cette idée est basée sur le double standard que j'ai évoqué tout à l'heure. La salope, c'est aussi «l'autre », la «mauvaise » femme, qu'il peut être stratégique de dénoncer afin de s'en démarquer. In fine, le concept est creux. Une salope, ça n'existe pas. C'est un mythe, érotique, grotesque et issu de l'imaginaire viril. En mystifiant une personne, en en faisant une espèce de monstre, une figure, on la prive de son identité et de son 
individualité. Ainsi réduites, les femmes (isolées, c'est un des effets de cette forme de stigmatisation) disparaissent. Pour ma part, je traduis slutshaming par «utilisation du concept de salope pour stigmatiser » et définis la salope, sur le modèle de Paola Tabet qui définit la prostituée par le stigmate dont elle est victime (Tabet 2004), à travers l'oppression qu'elle subit : une salope est une femme stigmatisée, point.

Qu'est-ce que le « viol moral »?

C'est une des femmes qui a témoigné lors d'une Marche qui a employé cette expression. Elle nous a expliqué que, quand ses partenaires lui demandaient son consentement pour une pratique sexuelle dont elle n'avait pas envie, elle n'était pas capable de dire NON, ce qui l'amenait à dire oui. Elle a explicité la difficulté qu'ont les femmes à se sentir légitimes à refuser. Plusieurs autres témoignages nous ont appris que les femmes se défendent rarement physiquement en cas d'agression sexuelle, et qu'elles n'expriment pas verbalement systématiquement leur refus. Il est évident que la question de la légitimité à exprimer son refus, à dire non, est centrale, et que cette légitimité est une construction sociale encore totalement biaisée par le double standard. C'est pourquoi, pour lutter contre les violences, il est impératif de lutter contre le slutshaming et repenser la légitimité. Il est indispensable de donner aux femmes les outils nécessaires à une approche agentive de leur sexualité et de leurs désirs.

Pourquoi se réapproprier le terme salope?

En 2011, j'ai appris que des femmes, à Toronto, se réclamaient du mot salope suite aux déclarations d'un policier dans un campus. Celui-ci s'était adressé aux femmes en disant : « Pour ne pas vous faire violer, évitez de vous habiller comme des salopes. » La logique de créer, suite à cette déclaration, la Marche des Salopes, m'a semblé évidente. C'était drôle, provocateur et plein de bon sens. Mais j'ai mis plusieurs années à formuler clairement l'architecture de ce bon sens.

On a beaucoup dit que les salopes se réappropriaient leur stigmate pour lui donner une autre valeur, un sens positif. Et on a beaucoup critiqué cette idée, car il est évident qu'un tel changement de sens est long et périlleux, et que sur la route, le public ne sera pas toujours prêt à suivre, que cela créera des zones de confusion, d'ambiguïté. Ces critiques sont fondées. Il est compliqué de reprendre un terme aussi chargé que le mot salope pour en faire un énoncé positif. Utiliser le mot salope n'équivaut pas seulement à vouloir changer sa signification. S'appeler la Marche des Salopes suite aux déclarations de ce policier, ou suite à toutes les utilisations négatives de ce mot, en particulier autour des cas de violences sexuelles, c'est avant tout une critique et une dénonciation. Plusieurs personnes interviewées dans un documentaire tourné par Anne-Claire Adet à propos de l'association en $2013^{4}$ ont parlé de « vulgarité » quant à l'usage du mot salope, or il est bien évident que la vulgarité est l'œuvre de ceux qui l'utilisent pour stigmatiser et non pas de celles qui remettent en question son usage à des fins militantes. On s'étonne souvent du fait qu'il semble davantage choquant que des femmes appellent un mouvement « la marche des salopes » plutôt que ce mot soit utilisé comme arme pour stigmatiser. La société patriarcale et hétérosexiste considère comme légitime le fait qu'on stigmatise une femme, notamment celles dont la sexualité est jugée déviante, mais ne supporte pas qu'on visibilise cette stigmatisation en remettant le stigmate en question. 
S'appeler soi-même une salope en militant contre les violences est assez efficace pour en dénoncer l'absurdité. Et les réactions vives qu'on rencontre montrent que cela provoque un réel trouble dans les esprits. Le fait que des femmes reprennent ellesmêmes ce mot à leur compte va à l'encontre de ce qui est socialement prescrit : s'en défendre. Or, premièrement, se défendre d'être une salope est totalement inutile, une fois qu'est posé le stigmate, car la résistance est contre-productive. S'agissant d'une attaque hétéronome et unilatérale, la personne stigmatisée ne fait pas le choix d'être insultée et n'a pas la possibilité de refuser l'insulte. Deuxièmement - et il s'agit là d'une posture militante - si on se défend d'être une salope, on entretient le fait qu'il existe bel et bien une réalité autour de ce mot, or nous l'avons vu, ce n'est pas le cas et le démontrer doit être une priorité. Le fait de s'affirmer salope avec fierté, c'est comme le dit Didier Éribon, «le processus de subjectivisation par lequel on passe du statut de stigmatisé à la revendication de soi-même, à la transformation de soi-même en sujet assumé de ses émotions, de ses désirs, de sa sexualité, de son rapport aux autres" (Éribon 2015: 19). Les symboles de saloperie (sluthood) sont mobilisés: mini-jupe, soutien-gorge, rouge à lèvres, etc., pour donner une identité imagée au groupe, et cette fabrication est artificielle, puisqu'il n'y a aucun comportement auquel se rattacher pour le définir. Les salopes sont éclatées par le stigmate, puisqu'un de ses effets est d'isoler les individus, il s'agit donc de faire groupe, symboliquement.

Quel est le rapport entre humour et slutshaming?

Comme beaucoup de formes de stigmatisation, le slutshaming s'appuie sur le sens commun. Le concept de salope n'est basé que sur des conventions sociales informelles, et malgré tout connues de toutes et tous. Sans appeler une femme salope, on peut facilement faire entendre que sa sexualité ou tout autre aspect de sa vie est " suspect ", et ce, de manière graveleuse et entendue. Il y a peu, alors que je travaillais à la radio et que par conséquent les auditeurs et auditrices ne pouvaient pas voir ma tenue, mes collègues journalistes et humoristes ont explicité le fait que j'étais en tenue " estivale » et que «quand il ferait plus chaud, on se demande ce que j'allais porter». Ici, aucune référence à ma sexualité ni même au mot salope, mais un ensemble de conventions communes, sur le ton de l'humour, pour me réduire à mon corps et à un aspect constitutif de la saloperie (sluthood), l'habillement jugé trop léger's. En l'occurrence il s'agit d'un exercice de joute verbale radiophonique: la pratique du slutshaming peut avoir comme objectif de déstabiliser et décrédibiliser une interlocutrice ou de faire rire le public et créer une connivence. Il n'empêche que cela formalise une hiérarchie entre les personnes qu'on peut discréditer sur la base de leur tenue (notamment) et ceux qui détiennent le pouvoir de les discréditer. D'autant que pour faire de l'humour, comme je l'ai écrit dans mon essai, il faut maîtriser la norme et les codes afin d'en jouer, la légitimité, le charisme, l'aisance dans la prise de parole et un certain capital culturel étant souvent les attributs du groupe dominant. Il s'agit bien, en fin de compte, de la reproduction de rapports de pouvoir. Le groupe dominé, dont la sexualité est le plus souvent vue comme dégradante et dégradée, est "au service du plaisir» du groupe dominant, qui en outre est considéré comme tenu de la protéger (face à des hommes qui souhaitent braver les barrières pour " arriver à leurs fins »). Ne pas être menacé de slutshaming et évoluer dans sa vie sexuelle sans contraintes est un privilège de dominant. 
Vous privilégiez la non-mixité, pourquoi?

Nous prônons et organisons des espaces de non-mixité militante. Par exemple, après la Marche, une soirée a été organisée. Cette soirée avait pour particularité une salle de danse, avec concerts et DJs, non mixte, réservée aux femmes ${ }^{6}$. Après cette soirée, une amie chercheuse m'a confié : «Vivre l'espace non mixte d'hier valait 60 discussions sur le sujet. » Libres des violences sexistes qui structurent complètement les espaces de vie nocturne, les femmes témoignent d'une expérience entièrement nouvelle qui leur permet de réaliser à quel point nos comportements sont dictés par l'omniprésence de ces violences quotidiennes. Cette non-mixité est utile, et justifiée par les oppressions que subissent certains groupes.

Le fait que le groupe soit composé principalement de femmes blanches universitaires et issues de milieux socio-économiques favorisés signifie-t-il qu'il est « limité »?

C'est le cas, et c'est très important de s'en faire la critique. L'histoire coloniale et les structures racistes qui perdurent ont produit des mythes érotisants autour des femmes racisées, spéculant sur leur sexualité débridée (et donc à contrôler), comme le montre Gail Pheterson (2001). Autrement dit les femmes racisées, en plus de subir du racisme, sont davantage exposées à la stigmatisation. Il est donc fondamental que la slutwalk parvienne à lutter contre le racisme, sans le reproduire. Pourtant pour l'instant, nous n'avons pas suffisamment mis nos intentions en action. Je voudrais citer Amandine Gay, réalisatrice : «Ce que j'observe c'est qu'il y a un souci à se penser soi-même pour les dominante's ou les gens qui appartiennent aux groupes majoritaires et donc moi je dirais par exemple que je n'aime pas le terme inclusivité et je pense que les Blanche's devraient d'abord réfléchir à la blanchité, à ce que ça veut dire d'être Blanche avant même de se poser la question d'“est-ce qu'il y a assez de personnes non blanches parmi nous ??" Demande-toi pourquoi elles ne viennent pas. » Et nous avons encore du travail.

Quelles voies de résistance encouragez-vous?

Les oppressions structurent les moyens de lutte. Les stigmates façonnent nos capacités et nos outils de résistance, nos armes et nos possibilités à faire groupe. L'ordre social établit des normes, hétérosexistes, racistes et bourgeoises, et met en place des signes permettant de hiérarchiser. Mais les identités sont multiples, les oppressions se croisent et se superposent. Les catégoriser est dangereux mais parfois nécessaire, pour les comprendre et y faire face spécifiquement.

En ce qui concerne le slutshaming, il est difficile de faire groupe, puisqu'il est quasiment impossible de s'identifier au stigmate avec fierté. Le slutshaming pousse les personnes stigmatisées, au contraire, à se distancer des autres salopes pour s'en démarquer, voire à performer la stigmatisation pour montrer que la salope c'est l'autre. Et comme nous l'avons vu, le slutshaming n'est basé sur aucun comportement précis et menace toutes les femmes. C'est donc une démarche féministe qui permet de le combattre. À partir du moment où toutes les femmes sont menacées par ce stigmate qui, de plus, les isole les unes des autres, il s'agit d'apprendre à faire groupe, et à développer une fierté. Non pas un groupe de "salopes" puisqu'une salope ça n'existe pas, mais de femmes qui s'unissent pour lutter contre la stigmatisation et qui se soutiennent activement les unes les autres. En faisant nombre, nous détruisons aussi le pouvoir marginalisant de l'insulte, car si toutes les femmes sont qualifiées de salopes, il n'y a plus de salopes, et le stigmate perd son pouvoir de faire la différence entre les femmes «bien » et celles qu'on peut disqualifier, violer, humilier... Nous n'avons pas choisi le terme de salope, il s'est imposé avec violence: on y fait face. Il s'agit finalement d'une stratégie 
d'autodéfense féministe. Face à une agression, ne pas rester silencieuse, mais agir et refuser la violence ${ }^{8}$.

\section{BIBLIOGRAPHIE}

Éribon, Didier, 2015, Une morale du minoritaire, Paris, Flammarion.

Greco, Luca, 2014, «La mise en scène d'une transition scientifique et identitaire : expérience narrative, identité et catégorisation », Semen, $n^{\circ} 37$, p. 113-125.

Mercier, Élisabeth, 2016, «Sexualité et respectabilité des femmes, la Slutwalk et autres (re)configurations morales, éthiques et politiques ", Nouvelles questions féministes, $\mathrm{n}^{\circ}$ 35, p. 16-47.

Pheterson, Gail, 2001, Le Prisme de la prostitution, édition augmentée de la version anglaise, trad. Nicole-Claude Mathieu, Paris, L'Harmattan.

Tabet, Paola, 2004, La Grande Arnaque. Sexualité des femmes et échange économico-sexuel, Paris, L'Harmattan.

Senarclens, Coline de, 2012, Putain de Militance, l'association Aspasie, un espace de mobilisation prostituée, 1982-1990, Genève, Université de Genève, travail de mémoire.

Senarclens, Coline de, 2014, Salope !, Vevey, Hélice Hélas Éditeur.

\section{NOTES}

1. https://www.facebook.com/slutwalksuisse/.

2. Témoignage disponible intégralement sur le site http://www.slutwalk.ch/, consulté le 2 juin 2016.

3. Voir le discours que j'ai rédigé pour la sortie de Salope ! en 2014 : «Une salope ça n'existe pas », http://sansdeclinersnarclens.tumblr.com/post/102688224263/une-salope-\%C3\%A7a-nexiste-pas, 2014, consulté le 2 juin 2016.

4. Anne-Claire Adet, "Salope en marche», 2013, disponible sur Vimeo: https:// vimeo.com/99828392.

5. Émission Les Beaux Parleurs, La Première, radio suisse romande, du dimanche 8 mai 2016.

6. Les personnes transgenre ( $\mathrm{M}$ to $\mathrm{F}$ et $\mathrm{F}$ to $\mathrm{M}$ ) sont bienvenu'e's dans nos événements non mixtes.

7. Entretien avec Amandine Gay, consulté sur https://renverse.co/Afrofeminisme-Entretienavec-Amandine-Gay-914.

8. À propos de l'autodéfense féministe, voir le «Non c'est Non» de Irène Zeilinger, disponible intégralement sur http://www.editions-zones.fr. 


\section{AUTEURS}

\section{STÉPHANIE PAHUD}

Université de Lausanne, École de français langue étrangère 